RECYT

Año 22 / No 33 / 2020 / 14-19

\title{
Optimización de las condiciones de extracción de un colorante natural a partir de Morus nigra
}

\section{Optimization of the extraction conditions of a natural dye from Morus nigra}

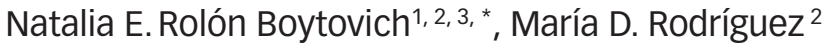 \\ 1- Facultad de Ciencias y Tecnología (FaCyT). Universidad Nacional de Itapúa (UNI). Abog. \\ Lorenzo Zacarías $\mathrm{N}^{\circ} 255 \mathrm{c} /$ Ruta $\mathrm{N}^{\circ} 1$. Encarnación, Itapúa, Paraguay.
}

2- Laboratorio de Biotecnología Molecular. Instituto de Biotecnología de Misiones. Facultad de Ciencias Exactas, Químicas y Naturales (FCEQyN). Universidad Nacional de Misiones (UNaM). Ruta Nacional No 12, Km 7,5. Posadas, Misiones, Argentina.

3- Laboratorio de Análisis de Agua y Alimentos de la Fundación de Desarrollo e Investigación Científica y Tecnológica (DINCYT). Av. Mitre N²2283. Posadas, Misiones, Argentina.

*E-mail: natyerb@gmail.com

\section{Resumen}

Recibido el 29 de abril de 2018, Aprobado el 8 de noviembre de 2019.

\begin{abstract}
El objetivo del presente trabajo fue optimizar el proceso de extracción de un producto concentrado a partir de Morus nigra para utilizarlo como colorante natural en alimentos. Los frutos de esta planta poseen antocianinas las cuales son de interés para la industria alimentaria. Se utilizó un diseño experimental para optimizar la extracción del colorante y los factores a evaluar fueron la concentración de etanol, la temperatura y el tiempo. Como resultado del diseño, los factores de relevancia resultaron ser la concentración de etanol y la temperatura, no así el tiempo de extracción. Así, se obtuvo una combinación de variables que maximizó su recuperación: concentración de etanol de $6,46 \% \mathrm{v} / \mathrm{v}$, temperatura de $75^{\circ} \mathrm{C}$ y un tiempo de $30 \mathrm{~min}$. Con estas condiciones óptimas, se podrán realizar extracciones de antocianinas, que generen mayores rendimientos, y, por lo tanto, puedan aplicarse como colorante alimenticio.
\end{abstract}

Palabras clave: Colorante alimenticio; Antocianinas; Morus nigra; Diseño factorial; Optimización.

\section{Abstract}

The aim of this research was to optimize the extraction process of a concentrated product from Morus Nigra to be later used as a natural food coloring. The fruits of this plant contain anthocyanins, which are of special interest within Food Industry. To improve dye extraction, an experimental design was used and the factors under evaluation were ethanol concentration, temperature and time. Consequently, ethanol concentration and temperature turned out to be the relevant factors; but not timing. This design resulted in a combination of variables that maximized dye recovery by obtaining $6.46 \%$ of ethanol concentration at 75 degrees centigrade within thirty-minute-time. These anthocyanin extractions generate optimal conditions and, therefore, can be applied as food coloring.

Keywords: Food coloring; Anthocyanins; Morus nigra; Factorial design; Optimization.

\section{Introducción}

El color en los alimentos es muy importante debido a que es el primer contacto que el consumidor tiene con ellos, por lo que es determinante para la aceptación o rechazo de los mismos [1], [2], y con frecuencia se lo utiliza como un índice de calidad, frescura y buen estado. Desafortunadamente, el color puede cambiar o perderse durante los procesos tecnológicos o el almacenamiento. Por esta razón, controlar, cambiar y estabilizar el color es uno de los principales objetivos de los científicos y los tecnólogos en la rama de los alimentos.

Los alimentos que no tienen color propio, como las golosinas, algunos postres, los snacks y las bebidas, entre otros; se colorean artificialmente para hacerlos más atractivos al consumidor.

Desde la aparición de los colorantes sintéticos en la industria, los colorantes naturales fueron relegados, ya que los primeros presentan mayor estabilidad y menor costo. Sin embargo, desde hace unos años la calidad y variedad de los colores provenientes de fuentes naturales ha mejorado notablemente, lo que ha permitido que su uso aumente progresivamente, sobre todo en la industria de los alimentos, ya que, en este campo, se buscan opciones cada vez más saludables y libres de compuestos químicos con potenciales efectos adversos sobre la salud. Se han 
desarrollado investigaciones en el campo de los colorantes enfocándose principalmente en lo relacionado con toxicidad, efectos secundarios, obtención de colorantes naturales y sustitución de los colorantes sintéticos [3].

Dentro de los pigmentos de origen natural, se hallan las antocianinas, que pueden obtenerse a partir de plantas $\mathrm{y}$ vegetales que constituyen una fuente importante de colorantes de la gama de los rojos y azules. El fruto de la planta Morus nigra es bien conocido como una buena fuente de antocianinas con diversas actividades biológicas [4]. Este fruto constituye una buena fuente de pigmentos rojos naturales, hidrosolubles, con potenciales usos en la industria de alimentos, donde existe una demanda considerable de colores naturales alternativos a los sintéticos, como el rojo $\mathrm{N}^{\circ} 40$ [5].

El interés por estos pigmentos antociánicos se da por sus posibles efectos terapéuticos y benéficos, dentro de los cuales se encuentran: prevención de enfermedades coronarias, efectos anticancerígenos, antitumorales, antiinflamatorios y antidiabéticos; además del mejoramiento de la agudeza visual y del comportamiento cognitivo. Las propiedades bioactivas de las antocianinas abren una nueva perspectiva para la obtención de productos coloreados con valor agregado para el consumo humano [5].

Por lo tanto, la extracción de estos pigmentos a partir de la mora es una alternativa comercial válida debido a que los pigmentos antociánicos, pueden ser un sustituto eficaz de los sintéticos, brindando a las personas mayor seguridad en los productos que consumen [6].

El objetivo de este trabajo fue extraer un colorante a partir de los frutos de Morus nigra, optimizando los parámetros que influyen en el proceso.

\section{Materiales y Métodos}

Obtención de la Muestra: Para la obtención de la muestra se siguió el plan de muestreo sugerido por la Norma Venezolana [7], correspondiente a la toma de muestra de frutas y hortalizas. Estuvieron conformadas por los frutos de la planta de Mora (Morus nigra), perteneciente a la familia de las moráceas. Fueron recolectadas manualmente de distintos árboles de la zona centro de Encarnación, Paraguay.

Se seleccionaron los frutos que se encontraban en buen estado, con la finalidad de obtener una muestra homogénea, descartando para ello los frutos en inmadurez fisiológica, no enteros, malformados, con presencia de daños por insectos - plagas, con deformaciones, roturas, rajaduras, raspaduras, manchados, podridos y con presencia de hongos [8]. En la figura 1 se esquematiza los pasos seguidos a lo largo de todo el experimento.

Trituración y secado: Los frutos se pesaron y trituraron con una procesadora de alimentos (Moulinex - slim force - 700W) hasta obtener una pasta, con el fin de generar mayor área superficial y así facilitar el secado. La pasta obtenida se secó en estufa a $60^{\circ} \mathrm{C}$ por $10 \mathrm{~h}$ para poder almacenarlas sin que se deterioren. En esta etapa se eliminó aproximadamente el $85 \%$ de humedad.

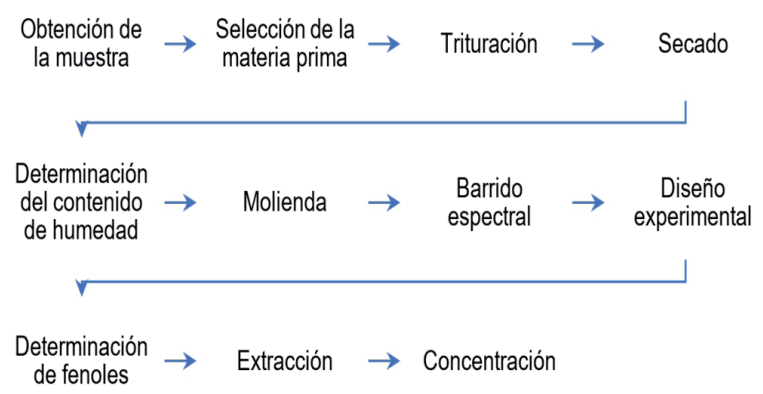

Figura $\mathbf{N}^{\circ} \mathbf{1}$ : Diagrama de flujo del experimento para la obtención de datos.

Determinación del contenido de humedad: Se pesaron $5 \mathrm{~g}$ de muestra seca y se colocaron en un humedímetro (KERN - MLS) a fin de determinar el porcentaje de hmedad presente. Esto se realizó por duplicado.

Molienda: Una vez seca la materia prima se procedió con la molienda del material, utilizando un mortero de porcelana. Esto se realizó para permitir aún mayor área de contacto entre el solvente (etanol en agua destilada a distintas concentraciones) y el material vegetal.

Barrido Espectral: Se ensayaron la concentración de solvente, temperatura y tiempo de extracción según los puntos centrales del diseño de experimentos; éstos fueron, etanol $40 \%$ a $53^{\circ} \mathrm{C}$ durante $45 \mathrm{~min}$. Se pesaron $0,5 \mathrm{~g}$ de frutos secos y molidos tal como se describe anteriormente y se colocaron en distintos tubos tipo Falcon de $15 \mathrm{~mL}$, se añadieron $10 \mathrm{~mL}$ de la concentración etanólica en cada tubo y se incubaron a las condiciones predichas. Una vez transcurrido el tiempo se procedió al centrifugado. Con el extracto obtenido se realizó un barrido espectral entre $200-700 \mathrm{~nm}$ a fin de determinar la longitud de onda de mayor absorbancia de las antocianinas; teniendo en cuenta dos bandas de absorción en la región UV $(260-280 \mathrm{~nm})$ y la región visible $(490-550 \mathrm{~nm})$ para este tipo de compuestos [9]. En base al barrido espectral se seleccionaron las longitudes de onda de mayor absorbancia para medir el contenido de antocianinas.

Diseño experimental: Para evaluar el efecto de la concentración de etanol, la temperatura y el tiempo en la extracción del colorante, a partir de un peso fijo de frutos de mora secos y molidos $(0,5395 \mathrm{~g})$, se ensayó cada variable independiente a 5 niveles (tabla 1). Las variables de respuesta fueron las absorbancias a las longitudes de onda de 285 y $525 \mathrm{~nm}$ y el contenido de fenoles totales.

Los ensayos se realizaron siguiendo un diseño de compuesto central $32+$ estrellas, definido mediante software estadístico Statgraphics Centurión XVI, con 17 corridas en total y 7 grados de libertad para el error. 
Determinación de fenoles: El contenido fenólico total se determinó utilizando el método espectrofotométrico de Folin-Ciocalteu [10] con modificaciones. Se realizó una curva de calibrado con patrones de concentración conocida a partir de una disolución madre de 900 ppm $(0,045 \mathrm{~g}$ de ácido gálico disuelto en $50 \mathrm{~mL}$ de agua destilada) y a partir de ella se prepararon las diferentes diluciones por duplicado.

Una vez preparadas las diluciones de la curva de calibrado, se añadió a todos los tubos $3 \mathrm{~mL}$ de agua destilada, $40 \mu \mathrm{L}$ del líquido correspondiente ya sea agua, patrón o muestra y $200 \mu \mathrm{L}$ del reactivo de Folin-Ciocalteu (Sigma - Aldrich) a todos los tubos, se homogenizó el contenido, se dejó reposar por 5 min y se añadió $600 \mu \mathrm{L}$ de $\mathrm{Na} 2 \mathrm{CO} 3$ (Merck) al 20\%, cerrando y agitando nuevamente. Se llevaron los tubos a $40^{\circ} \mathrm{C}$ durante $30 \mathrm{~min}$ y, una vez fríos, se midió la absorbancia a $765 \mathrm{~nm}$. El contenido total de fenoles se expresó como equivalente de ácido gálico GAE/g.

Extracción: Se pesaron $35 \mathrm{~g}$ de mora seca y molida, se agregaron a una solución de $700 \mathrm{~mL}$ de etanol - agua al $6,43 \% \mathrm{v} / \mathrm{v}$, y se llevó a $75^{\circ} \mathrm{C}$ en baño termostático durante $30 \mathrm{~min}$. Seguidamente se filtró y lo obtenido se guardó en un frasco color caramelo para evitar así su exposición a la luz y al oxígeno.

Concentración: Se realizó en un evaporador rotatorio al vacío marca SENCO operando a $60^{\circ} \mathrm{C}$ y 0,8 atm durante 2 $\mathrm{h}$ aproximadamente, obteniéndose $50 \mathrm{~mL}$ de concentrado.

Validación: Se hicieron extracciones, por triplicado, bajo las condiciones predichas como óptimas, según las gráficas de superficie de respuesta, siendo éstas para 285 nm: $6,46 \%$ de concentración de etanol a $75,09^{\circ} \mathrm{C}$ y un tiempo de $30 \mathrm{~min}$; para $525 \mathrm{~nm}$ : la misma cantidad de tiempo, pero a $55,6^{\circ} \mathrm{C}$ y $0 \%$ de etanol; y para los fenoles totales una concentración de etanol de $44,5 \%$, a la temperatura de $80^{\circ} \mathrm{C}$ y un tiempo de extracción $30 \mathrm{~min}$. Se procedió a determinar las absorbancias y el contenido de fenoles totales.

\section{Resultados y Discusión}

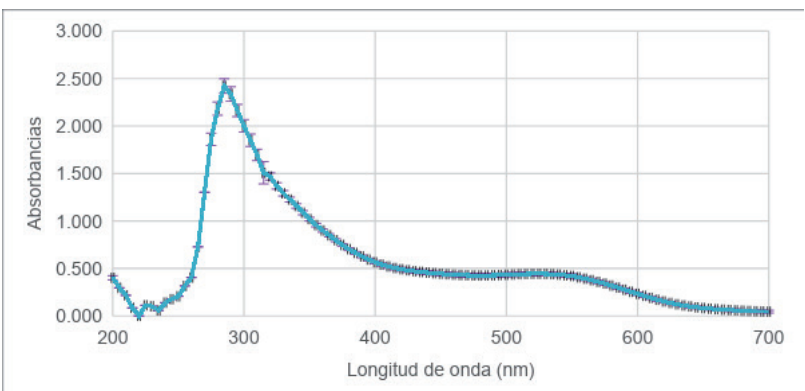

Figura 2: Barrido espectral para detectar picos de máxima absorción de antocianinas en Morus nigra.

Barrido Espectral: Las muestras presentaron una humedad de 7,92 $\pm 0,01$. El contenido de agua de las muestras fue tomado en cuenta para los cálculos de la cantidad de agua dependiendo de la concentración de solvente en las extracciones. Se procedió al barrido espectral entre los 200 y $700 \mathrm{~nm}$ a fin de determinar las longitudes de onda de mayor absorbancia de las antocianinas. Las longitudes de onda seleccionadas fueron de 285 y $525 \mathrm{~nm}$, lo cual concuerda satisfactoriamente con datos teóricos confirmados en otros estudios, en donde se describe un pico máximo de absorción de $285 \mathrm{~nm}$ para piranoantocianinas [11] y $525 \mathrm{~nm}$ para antocianinas [12], tal como se muestra en la figura 2.

Diseño Experimental: Para establecer la influencia de las variables utilizadas sobre el proceso de extracción del colorante se realizaron una serie de ensayos siguiendo el diseño de experimentos, basado en un diseño compuesto central 32 + estrellas, definido mediante el software estadístico Statgraphics Centurión XVI. Los resultados experimentales obtenidos al realizar las mediciones de las absorbancias de los extractos utilizando dicho diseño se presentan en la tabla 1; la cual contiene los promedios de las absorbancias y el contenido fenólico.

Tabla 1: Diseño compuesto central 32 + estrellas para evaluar la concentración de etanol, la temperatura y el tiempo de extracción de antocianinas de Morus nigra.

\begin{tabular}{|c|c|c|c|c|c|c|}
\hline Corrida & \% Etanol & Temperatura & Tiempo & $\begin{array}{c}\text { Abs } \\
\mathbf{2 8 5 n m}\end{array}$ & $\begin{array}{c}\text { Abs } \\
\mathbf{5 2 5 n m}\end{array}$ & $\begin{array}{c}\text { Fenoles } \\
\text { (GAE/g) }\end{array}$ \\
\hline 1 & 16 & 70 & 54 & 0,653 & 0,433 & 1,170 \\
\hline 2 & 0 & 53 & 45 & 0,599 & 0,453 & 1,106 \\
\hline 3 & 40 & 53 & 30 & 0,579 & 0,41 & 1,406 \\
\hline 4 & 16 & 70 & 36 & 0,739 & 0,556 & 1,518 \\
\hline 5 & 64 & 36 & 36 & 0,517 & 0,351 & 1,160 \\
\hline 6 & 64 & 70 & 36 & 0,585 & 0,336 & 1,379 \\
\hline 7 & 64 & 36 & 54 & 0,485 & 0,316 & 1,068 \\
\hline 8 & 16 & 36 & 36 & 0,603 & 0,48 & 1,213 \\
\hline 9 & 64 & 70 & 54 & 0,662 & 0,459 & 1,483 \\
\hline 10 & 40 & 25 & 45 & 0,371 & 0,294 & 1,267 \\
\hline 11 & 40 & 80 & 45 & 0,619 & 0,429 & 1,313 \\
\hline 12 & 16 & 36 & 54 & 0,562 & 0,451 & 1,384 \\
\hline 13 & 40 & 53 & 60 & 0,622 & 0,461 & 1,541 \\
\hline 14 & 80 & 53 & 45 & 0,300 & 0,259 & 0,985 \\
\hline 15 & 40 & 53 & 45 & 0,625 & 0,395 & 1,259 \\
\hline 16 & 40 & 53 & 45 & 0,585 & 0,483 & 1,342 \\
\hline 17 & 40 & 53 & 45 & 0,584 & 0,433 & 1,554 \\
\hline & Análisis de varianza para las distintas variables de respuesta & \\
\hline
\end{tabular}

Se realizó el análisis de varianza para la concentración de etanol, la temperatura y el tiempo de extracción sobre las antocianinas observadas a $285 \mathrm{~nm}$. La concentración de etanol y la temperatura tuvieron un valor-p menor que 0,05 ; indicando esto que son estadísticamente significativos con un nivel de confianza del $95,0 \%$.

Para las antocianinas detectadas a $525 \mathrm{~nm}$, la concentración de etanol tuvo un valor-p menor que 0,05 ; indicando que es estadísticamente significativo sobre la variable respuesta, con un nivel de confianza del $95 \%$, resultando no significativas la temperatura y el tiempo.

El análisis de varianza para fenoles totales indicó que la concentración de etanol en su expresión cuadrática tuvo un valor-p menor que 0,05 ; indicando que es estadísticamente significativa, con un nivel de confianza del $95 \%$, resultando 
no significativas la temperatura y el tiempo.

En el diagrama de Pareto, figura 3, se muestran los gráficos de Pareto para el diseño estadístico.
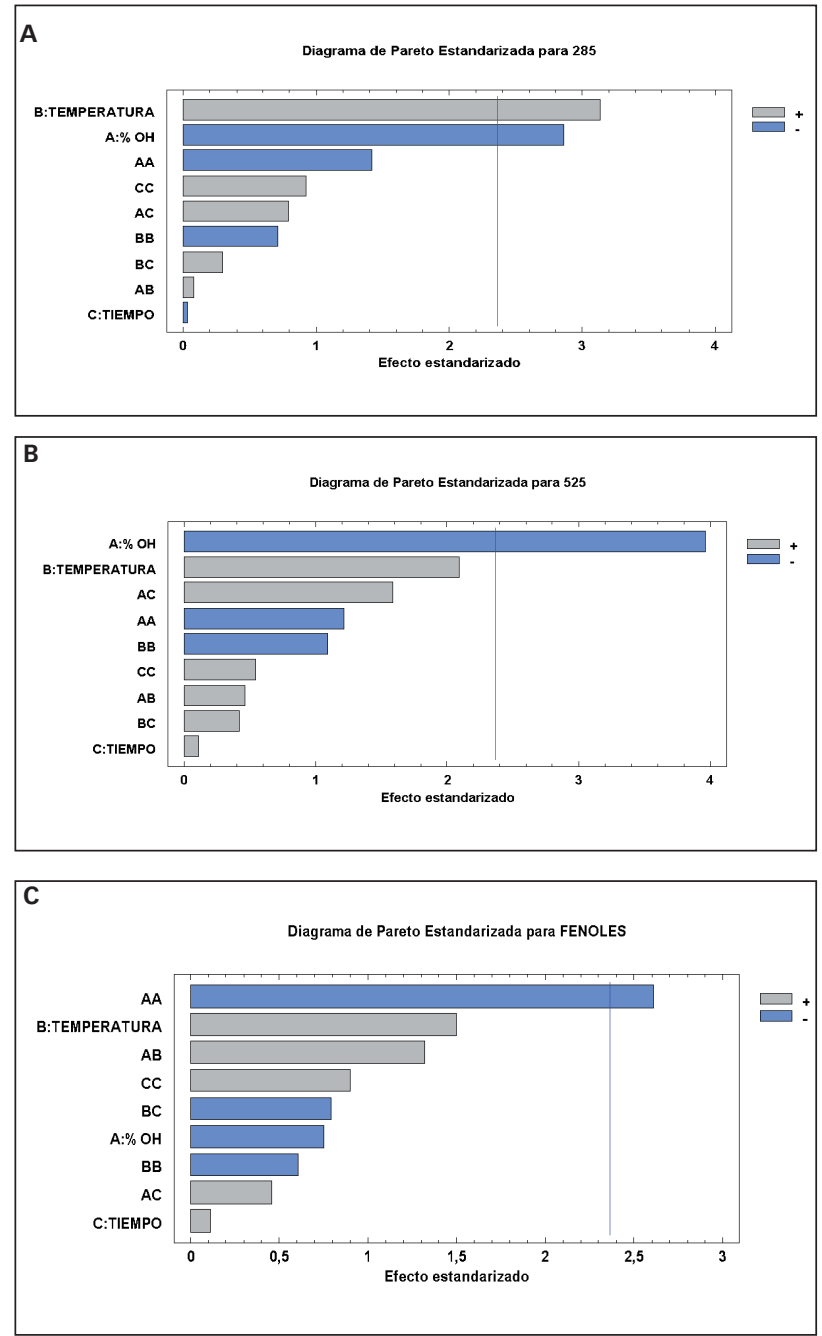

Figura 3: Diagrama de Pareto estandarizado para las respuestas A) para las antocianinas detectadas a $285 \mathrm{~nm}, \mathrm{~B}$ ) para las antocianinas detectadas a $525 \mathrm{~nm}$ y C) para los fenoles totales, extraídas de Morus nigra.

Como se puede corroborar en la (figura $3 \mathrm{~A}$ ), la temperatura y la concentración de etanol influyen tanto positiva como negativamente en la absorbancia a $285 \mathrm{~nm}$ del colorante, respectivamente. En cambio, en la absorbancia a $525 \mathrm{~nm}$ sólo la concentración de etanol tuvo un efecto negativo estadísticamente significativo, (figura $3 \mathrm{~B}$ ). Respecto a los fenoles totales (figura $3 \mathrm{C}$ ), la concentración de etanol en su expresión cuadrática tuvo efecto negativo estadísticamente significativo. Un factor cuadrático negativo y estadísticamente significativo, indica que la variable respuesta disminuyó cuadráticamente, a medida que el nivel del factor se incrementaba [13].

En base a las corridas del diseño experimental y los datos obtenidos de las variables respuesta, se ajustó un modelo para cada una de las variables (ecuaciones 1, 2 y 3). Estas ecuaciones permiten calcular cada variable de respuesta en función de las variables independientes (concentración de etanol, la temperatura y el tiempo), e indican de qué manera se comportaría cada una de las respuestas manipulando dichas variables.

Abs285 $=0,592-0,059 * \mathrm{OH}+0,065 * \mathrm{~T}-0,0007 * \mathrm{t}-$ $0,032 * \mathrm{OH}^{2}+0,002 * \mathrm{OH}^{*} \mathrm{~T}+0,021 * \mathrm{OH}^{*} \mathrm{t}-0,016^{*} \mathrm{~T}^{2}+$ $0,008 * \mathrm{~T} * \mathrm{t}+0,02 * \mathrm{t}^{2}(1)$

$\operatorname{Abs525}=0,434-0,057 * \mathrm{OH}+0,030 * \mathrm{~T}+0,001 * \mathrm{t}-$ $0,019 * \mathrm{OH}^{2}+0,009 * \mathrm{OH}^{*} \mathrm{~T}+0,03 * \mathrm{OH}^{*} \mathrm{t}-0,017 * \mathrm{~T}^{2}+$ $0,008 * \mathrm{~T} * \mathrm{t}+0,009 * \mathrm{t}^{2}(2)$

Fenoles $(\mathbf{g} / \mathbf{L})=1,382-0,029 * \mathrm{OH}+0,059 * \mathrm{~T}+$ $0,005 * \mathrm{t}-0,112 * \mathrm{OH}^{2}+0,068 * \mathrm{OH}^{*} \mathrm{~T}+0,024 * \mathrm{OH}^{*} \mathrm{t}-$ $0,026 * \mathrm{~T}^{2}-0,040 * \mathrm{~T} * \mathrm{t}+0,039 * \mathrm{t}^{2}(3)$

Donde:

T: Temperatura $\left({ }^{\circ} \mathrm{C}\right)$

t: Tiempo (min)

$\mathrm{OH}$ : Concentración de etanol (\%)

A partir de las ecuaciones (1), (2), y (3); se analizó la influencia de las variables de proceso estudiadas. Para ello, se elaboraron diferentes gráficos de superficie de respuesta (figura 4). Dado que se trabajó con tres factores (concentración de etanol, temperatura, y tiempo de extracción) y tres variables de respuestas (absorbancias a $285 \mathrm{~nm}$, absorbancias a $525 \mathrm{~nm}$ y fenoles totales), los gráficos se obtuvieron manteniendo uno de los factores constantes en el punto central y variando los otros dos.

Se puede observar que, para la interacción entre la concentración de etanol y la temperatura de extracción, el máximo en la variable respuesta se obtuvo en un rango cercano para ambas absorbancias, sin embargo, se observa también un desplazamiento a niveles mayores en la superficie correspondiente a fenoles totales. Resultados similares se observaron en la interacción entre el tiempo y la temperatura de extracción, donde las gráficas para ambas absorbancias son similares y la zona con mayor valor de la variable respuesta corresponde a los niveles altos de ambas variables. Para fenoles totales, sin embargo, los valores máximos corresponden a niveles altos de temperatura y bajos de tiempo de extracción. En la tabla 5 se observan los valores óptimos de cada variable estudiada según las gráficas de superficie de respuesta.

Tabla 2: Valores óptimos para maximizar la obtención de antocianinas en extractos obtenidos a partir de Morus nigra.

\begin{tabular}{|c|c|c|c|c|c|}
\hline Factores & \multicolumn{2}{|c|}{ Niveles } & \multicolumn{3}{c|}{ Valores óptimos } \\
\hline & Bajo & Alto & $\mathbf{2 8 5} \mathbf{~ n m}$ & $\mathbf{5 2 5} \mathbf{~ n m}$ & Fenoles totales \\
\hline Etanol (\%) & 0 & 80 & 6,46 & 0 & 44,8 \\
\hline Temp. $\left({ }^{\circ} \mathrm{C}\right)$ & 25 & 80 & 75,09 & 55,6 & 80 \\
\hline Tiempo (min) & 30 & 60 & 30 & 30 & 30 \\
\hline
\end{tabular}

Validación: Para validar el modelo y el resultado máximo obtenido, se realizó una nueva prueba con 3 repeticiones usando los valores obtenidos en la tabla 2 , siendo éstas para $285 \mathrm{~nm} ; 6,46 \%$ de concentración de etanol a $75,09^{\circ} \mathrm{C}$ y un tiempo de $30 \mathrm{~min}$; para $525 \mathrm{~nm}$, la misma cantidad de tiempo pero a $55,6^{\circ} \mathrm{C}$ y $0 \%$ de etanol; y para los fenoles totales una concentración de etanol de 44,8\%, 

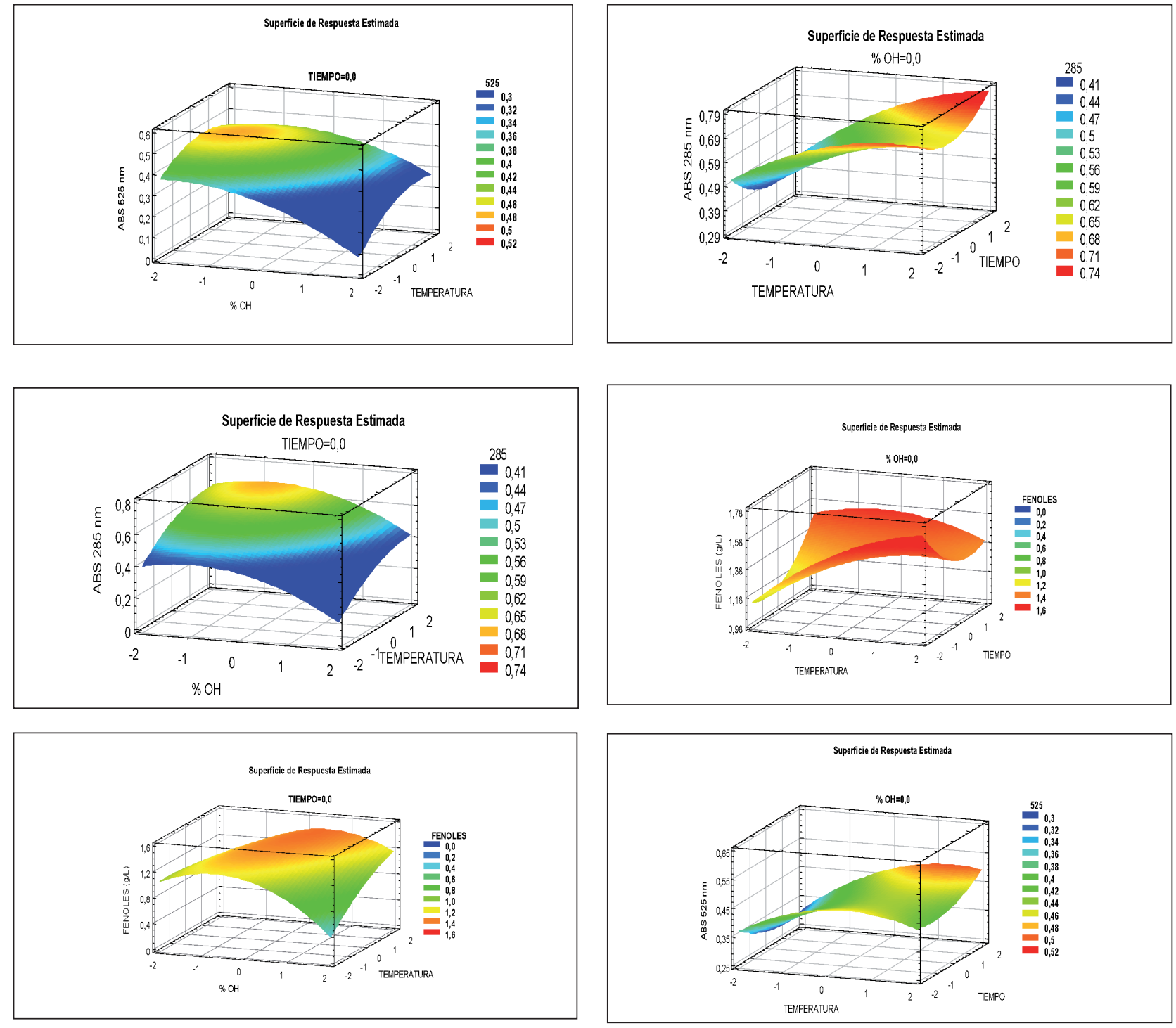

Figura 4: Superficie de respuesta estimada para la absorbancia a $285 \mathrm{~nm}$, a $525 \mathrm{~nm}$ y fenoles totales de extractos obtenidos a partir de Morus nigra.

a la temperatura de $80^{\circ} \mathrm{C}$ y un tiempo de extracción 30 min. La validación dió como resultado los valores que se muestran en la tabla 6 y que se comparan con los óptimos teóricos proyectados según las gráficas de superficie de respuesta para todas las condiciones.

Tabla 6: Comparación de valores óptimos teóricos y experimentales de las antocianinas detectadas y los fenoles totales para extractos de Morus nigra.

\begin{tabular}{|c|c|c|}
\hline Condiciones & Valor teórico & Valor experimental \\
\hline $285 \mathrm{~nm}$ & 0,759 & $0,642 \pm 0,083$ \\
\hline $525 \mathrm{~nm}$ & 0,582 & $0,157 \pm 0,301$ \\
\hline Fenoles totales (GAE/g) & 1,629 & $1,581 \pm 0,034$ \\
\hline
\end{tabular}

Con respecto a la concentración de etanol, las condiciones óptimas fueron de 6,46 y $0 \%$ para ambas longitudes de onda estudiadas y coinciden con el trabajo realizado por Vicente Castillo [14], en donde realizaron extracciones de antocianinas con distintos solventes, siendo el etanol el extractante menos apropiado.
Por su parte, en la optimización de las condiciones de extracción de antocianinas de batata morada, autores lograron la mejor extracción a $80^{\circ} \mathrm{C}$ [15]; mientras que otros autores, al estudiar la extracción de anocianinas a partir de maíz morado, obtuvieron que la mejor temperatura de extracción fue $75^{\circ} \mathrm{C}[16]$; coinicidiendo dichas temperaturas con este trabajo para las antocianinas medidas a $285 \mathrm{~nm}$ y los fenoles totales.

El tiempo de $30 \mathrm{~min}$, coincide con lo predicho por Markakis que recomienda utilizar altas temperaturas y cortos tiempos de procesamiento para una mejor retención del pigmento [17].

Mediante este diseño se pudo determinar que para la extracción del colorante los factores de relevancia resultaron ser la concentración de etanol y la temperatura, no así el tiempo de extracción. 


\section{Conclusión}

Mediante el empleo de diseños experimentales se pudo estudiar diferentes variables, analizar sus interacciones, y establecer cuál es la que influye significativamente sobre la extracción de antocianinas de Morus nigra. Se observó que los factores de relevancia resultaron ser la concentración de etanol y la temperatura, no así el tiempo de extracción. Con estas condiciones óptimas, se podrán realizar extracciones de antocianinas, que generen mayores rendimientos, $y$, por lo tanto, puedan aplicarse como colorante alimenticio.

\section{Agradecimientos}

Al Consejo Nacional de Ciencias y Tecnología (CONACYT) del Paraguay, por la beca otorgada para la realización de esta investigación y la financiación del mismo, también a la Facultad de Ciencias y Tecnología de la Universidad Nacional de Itapúa y al Instituto de Biotecnología de Misiones - Argentina por las instalaciones y los equipos otorgados.

\section{Referencias}

1. S. Badui Dergal. Química de los alimentos. 2006.

2. J. M. Espinosa. La ciencia sensorial: su incidencia en la calidad del servicio de alimentos y bebidas y la satisfacción del cliente, pp. 1-2, 2014.

3. M. Restrepo Gallego. Sustitución de colorantes en alimentos, Lasallista Investig., vol. 4, n.o 1, pp. 35-39, 2007.

4. P. Aramwit, N. Bang, у т. Srichana. The properties and stability of anthocyanins in mulberry fruits, Food Res. Int., vol. 43, n.o 4, pp. 1093-1097, 2010.

5. G. A. Garzón. Anthocyanins as natural colorants and bioactive compounds: A review. Acta Biológica Colombiana, vol. 13, n.o 3, pp. 27-36, 2008.

6. M. S. Butt, A. Nazir, M. T. Sultan, y K. Schroën. Morus alba L. nature's functional tonic, vol. 19, 2008.
7. COVENIN N 1769. Norma Venezolana. Frutas. Toma de Muestra, 1981. [En línea]. Disponible en: http://www. sencamer.gob.ve/sencamer/normas/1769-81.pdf. [Accedido: 24-ago-2017].

8. A. D. García Méndez. Evaluación de un Tratamiento postcosecha de la Tecnología IV Gama en Frutos de Moras (Rubus glaucus Benth), Rev. Iber. Tecnol. Postcosecha, vol. 9, n.o 1, pp. 44-54, 2008.

9. G. A. López Valdez. Extracción y Caracterización del Colorante del Fruto Almendra de la India (Terminalia catappa), Universidad Autónoma Agraria, 2012.

10. E. García Martínez, I. Fernández Segovia, y A. Fuentes López. Determinación de polifenoles totales por el método de Folin-Ciocalteu, Valencia - España, 2015.

11. A. Castañeda Ovando, J. A. Rodríguez, C. A. Galán Vidal, y S. Sánchez Herrera. Estudio de efectos que afectan la estabilidad de antocianinas y piranoantocianinas en solución acuosa mediante herramientas estadisticas, pp. 177-182, 2008.

12. M. Aguilera ortíz et al. Propiedades funcionales de las antocianinas, vol. 13, n.o 2, pp. 16-22, 2011.

13. M. D. Rodríguez et al. Obtención de azúcares fermentables a partir de aserrín de pino pretratado secuencialmente con ácido-base, Rev. Int. Contam. Ambient., vol. 33, n.o 2, pp. 317-324, 2017.

14. A. Vicente Castillo. Análisis de polifenoles en smoothies, zumos y néctares de frutas rojas. Universidad Politécnica de Cartagena, 2013.

15. G. Fan, Y. Han, Z. Gu, y D. Chen. Optimizing conditions for anthocyanins extraction from purple sweet potato using response surface methodology (RSM). LWT Food Sci. Technol., vol. 41, n.o 1, pp. 155-160, 2008.

16. A. Gorriti et al. Extracción de antocianinas de las corontas de las corontas de Zea mays L. "Maíz morado", Cienc. Invest., vol. 12, n.o 2, pp. 64-74, 2009.

17. P. Markakis. Anthocyanins as Food Additives. en Anthocyanins As Food Colors, Elsevier Science, 1982, pp. 245-253. 